\title{
Combination therapies to inhibit the RAF/MEK/ERK pathway in melanoma: we are not done yet
}

\author{
Grant A. McArthur ${ }^{1,2,3,4,5,6 *}$ \\ ${ }^{1}$ Department of Cancer Medicine, Peter MacCallum Cancer Centre, East Melbourne, VIC, Australia, ${ }^{2}$ Department of Pathology, \\ University of Melbourne, Parkville, VIC, Australia, ${ }^{3}$ Department of Medicine, St Vincent's Hospital, University of Melbourne, \\ Fitzroy, VIC, Australia, ${ }^{4}$ Sir Peter MacCallum Department of Oncology, University of Melbourne, East Melbourne, VIC, Australia, \\ ${ }^{5}$ Molecular Oncology Laboratory, Oncogenic Signaling and Growth Control Program, Peter MacCallum Cancer Centre, East \\ Melbourne, VIC, Australia, ${ }^{6}$ Translational Research Laboratory, Cancer Therapeutics Program, Peter MacCallum Cancer \\ Centre, East Melbourne, VIC, Australia
}

Keywords: BRAF, MEK, ERK, CDK4, apoptosis, BIM, melanoma

\section{OPEN ACCESS}

Edited by:

Ruggero De Maria,

Istituto Superiore di Sanità, Italy

Reviewed by:

Ryan J. Sullivan,

Massachusetts General Hospital

Cancer Center, USA

Piro Lito,

Memorial Sloan Kettering Cancer

Center, USA

Keiran Smalley,

Moffitt Cancer Center, USA

${ }^{*}$ Correspondence:

Grant A. McArthur

grant.mcarthur@petermac.org

Specialty section:

This article was submitted to Cancer Molecular Targets and Therapeutics, a section of the journal

Frontiers in Oncology

Received: 12 April 2015

Accepted: 03 July 2015

Published: 17 July 2015

Citation:

McArthur GA (2015)

Combination therapies to inhibit the RAF/MEK/ERK pathway in melanoma: we are not done yet. Front. Oncol. 5:161. doi: 10.3389/fonc.2015.00161

\section{The Combination of BRAF and MEK Inhibition in Advanced Melanoma}

The discovery and development of small molecule inhibitors of mutant BRAF kinase and MEK kinase have revolutionized the care of patients with melanoma. When used as single agents, the BRAF inhibitors, vemurafenib and dabrafenib, improve progression free and overall survival when compared to chemotherapy with dacarbazine $(1,2)$. The MEK inhibitor, trametinib, also improves progression-free survival compared to chemotherapy, but achieves a lower response rate at its maximum tolerated dose in a continuous schedule when compared to BRAF inhibitors (3). Therefore, single agent trametinib is generally reserved for patients with intolerance to BRAF inhibitors, an uncommon event.

The identification of mechanisms of resistance to single agent BRAF inhibitors that reactivate the RAF/MEK/ERK pathway (4-6) led to the hypothesis that addition of a MEK inhibitor to a BRAF inhibitor may prevent or delay the emergence of resistance. This has indeed been the case. Three separate phase 3 trials have shown superiority of dual BRAF and MEK inhibition when compared to single agents BRAF inhibition alone (7-9). Dabrafenib combined with trametinib was superior to dabrafenib alone or vemurafenib alone, and vemurafenib and cobimetinib were superior to vemurafenib alone with hazard ratios for progression-free survival favoring the combination arms of $0.75,0.56$, and 0.51 , respectively. Moreover, overall survival was also improved with hazard ratios, favoring the combination arms of $0.63,0.69$, and 0.65 , respectively. Interesting combination therapy also substantially improved the complete response rate from $4-9$ to $10-13 \%$ across the three studies.

In each of these three trials, it was also clear that combination therapy reduced the frequency of cutaneous side effects that have been attributed to BRAF inhibitor-induced "paradoxical activation" of RAF kinases in cells without BRAF mutations. This exactly predicts the findings from preclinical studies where MEK inhibition downstream of activated RAF kinases reduces signaling through ERK and the outputs of paradoxical activation (10). Overall, the combination of BRAF and MEK inhibition is remarkably well tolerated, alleviating the fear that combinations of signaling inhibitors, particularly those that target the same pathway, would not be tolerable. This is probably due to paradoxical activation of RAF kinases reducing the effects of MEK inhibition, and MEK inhibition reducing the effects of paradoxical activation of RAF kinases. 


\section{Mechanism of Resistance to Combined BRAF and MEK Inhibition}

Although the combined use of BRAF and MEK inhibition has clearly become a new standard for inhibiting the RAF/MEK/ERK pathway in patients with advanced BRAF mutant melanoma the problem of acquired resistance has become a major stumbling block to obtaining long-term disease control. In contrast, primary or innate resistance is very uncommon (7-9), suggesting that without the selective pressure of long-term pathway inhibition most melanoma cells with BRAF mutations are sensitive to RAF/MEK/ERK pathway inhibition.

A systematic approach using next generation sequencing of the exome of melanoma tissue from patients with acquired resistance has been employed by the Garraway, Lo, and Rizos groups to examine sequence variants of higher frequency in samples derived from lesions refractory to single agent BRAF inhibitors. Furthermore, RNA sequencing or reverse transcriptase-PCR has been used to examine the emergence of splice variants of BRAF that induce resistance to BRAF inhibition. With over 100 patient samples analyzed, a very clear pattern emerges with approximately two-thirds of patients acquiring genomic events that reactivate RAF/MEK/ERK signaling $(5,6,11-16)$. This correlates well with immunohistochemistry and reverse phase protein array studies that show a similar fraction of samples from patients with resistance to BRAF inhibitors with elevated phosphorylation of ERK in samples consistent with reactivation of RAF/MEK/ERK signaling $(17,18)$. One-third of patients were predicted to have mechanisms that bypass the requirement for reactivation of RAF/MEK/ERK signaling.

Interestingly, the three most common molecular events reactivating RAF/MEK/ERK signaling, BRAF amplification, NRAS mutations, BRAF splice variants, all promote dimerization of mutant BRAF with CRAF or wild type BRAF. Both vemurafenib and dabrafenib inhibit mutant BRAF by binding to the ATP binding pocket that in the setting of mutant BRAF monomers potently inhibit RAF/MEK/ERK signaling. However, in the setting of upstream activation of RAS (e.g., mutant NRAS), or amplified BRAF or truncated mutant BRAF generated by the splice variant, paradoxical activation of the pathway is induced through generation of RAF dimers (19-22). Furthermore, even in the absence of these genomic variants, BRAF inhibitors can enhance upstream activation of RAS through feedback mechanisms, resulting in a new adapted steady state of active MEK and ERK (23-26). In all these settings, inhibition of MEK and/or ERK should reduce pathway output consistent with the clinical outcomes of patients treated with combined BRAF and MEK inhibition.

Given improved clinical outcomes of combined BRAF and MEK inhibition and frequent reactivation of RAF/MEK/ERK signaling as a mechanism of resistance to BRAF inhibitor monotherapy, there is immense interest in understanding the mechanisms of resistance to combined BRAF and MEK inhibition. Because the combination of BRAF and MEK inhibition may more effectively inhibit RAF/MEK/ERK signaling one may predict a priori two possible outcomes: first, with more effective inhibition of RAF/MEK/ERK signaling, there would be an increase in the frequency of bypass mechanisms independent of RAF/MEK/ERK signaling given a higher threshold to reactivate RAF/MEK/ERK signaling; or second, with more effective inhibition of RAF/MEK/ERK signaling, it would be advantageous, or even essential to reactivate RAF/MEK/ERK signaling by further genomic events for cell proliferation to occur.

Studies from the Garraway, Lo, Rizos, and Chin groups clearly show the acquisition of sequence variants activating $\mathrm{RAF} / \mathrm{MEK} / \mathrm{ERK}$ signaling in patients developing resistance to combined BRAF and MEK inhibition (18, 27-29). These genomic events again include BRAF amplification, NRAS mutations, BRAF splice variants, and MEK mutations. If such studies are confirmed on larger datasets, this sets up the intriguing concept that as the $\mathrm{RAF} / \mathrm{MEK} / \mathrm{ERK}$ pathway is inhibited more effectively it becomes essential for a cell to overcome this inhibition if proliferation is to occur. Therefore, it follows that if the pathway can be even more effectively inhibited one may be able to raise the threshold for genomic events to reactivate the pathway so high that the frequency of acquired resistance could be dramatically reduced.

\section{Consequences of More Effective Inhibition of the RAF/MEK/ERK Pathway}

Inhibition of RAF/MEK/ERK signaling in melanoma cells with BRAF mutations results in cell cycle arrest and promotion of cell death, including apoptosis. Clinically, this manifests in reduced size of tumor masses, which is partial or even complete response. In support of this concept, there was a correlation between inhibition of phosphorylation or ERK and reduction in tumor size in patients treated with vemurafenib (30). Moreover, as mentioned above, combined BRAF and MEK inhibition increase the frequency of complete responses. However, it is worth considering the consequences of pathway inhibition in more detail. First, pathway inhibition can result in cells adapting to the inhibition of signaling with the acquisition of mesenchymal phenotype with enhanced cell migratory capacity and a change in cell metabolism (31-34). This allows cells to survive and potentially enables subsequent outgrowth of resistant cells. Second, the tumor microenvironment must change with therapy. There is a change in the leukocytic content of tumors (35-37), tumors contain dead and dying cells and some cells may acquire senescence-like features (38). All these factors may influence whether a cell capable of generating acquired resistance survives, dies, or is enforced into a non-proliferative state that maybe long term.

As summarized in Figure 1, enhanced inhibition of the RAF/MEK/ERK pathway may lead to more cell death or even a change in tumor microenvironment that is less compatible with long-term cell survival or the reacquisition of a proliferative state. This hypothesis remains speculative; however, the increased proportion of patients achieving complete response with combined BRAF and MEK inhibition, and the excellent survival of patients who achieve a complete metabolic response on FDG-PET scan (39), that is, a surrogate of inhibition of the RAF/MEK/ERK pathway (40), suggest that more effective or complete inhibition of RAF/MEK/ERK signaling may indeed produce biological responses that improve overall survival. 


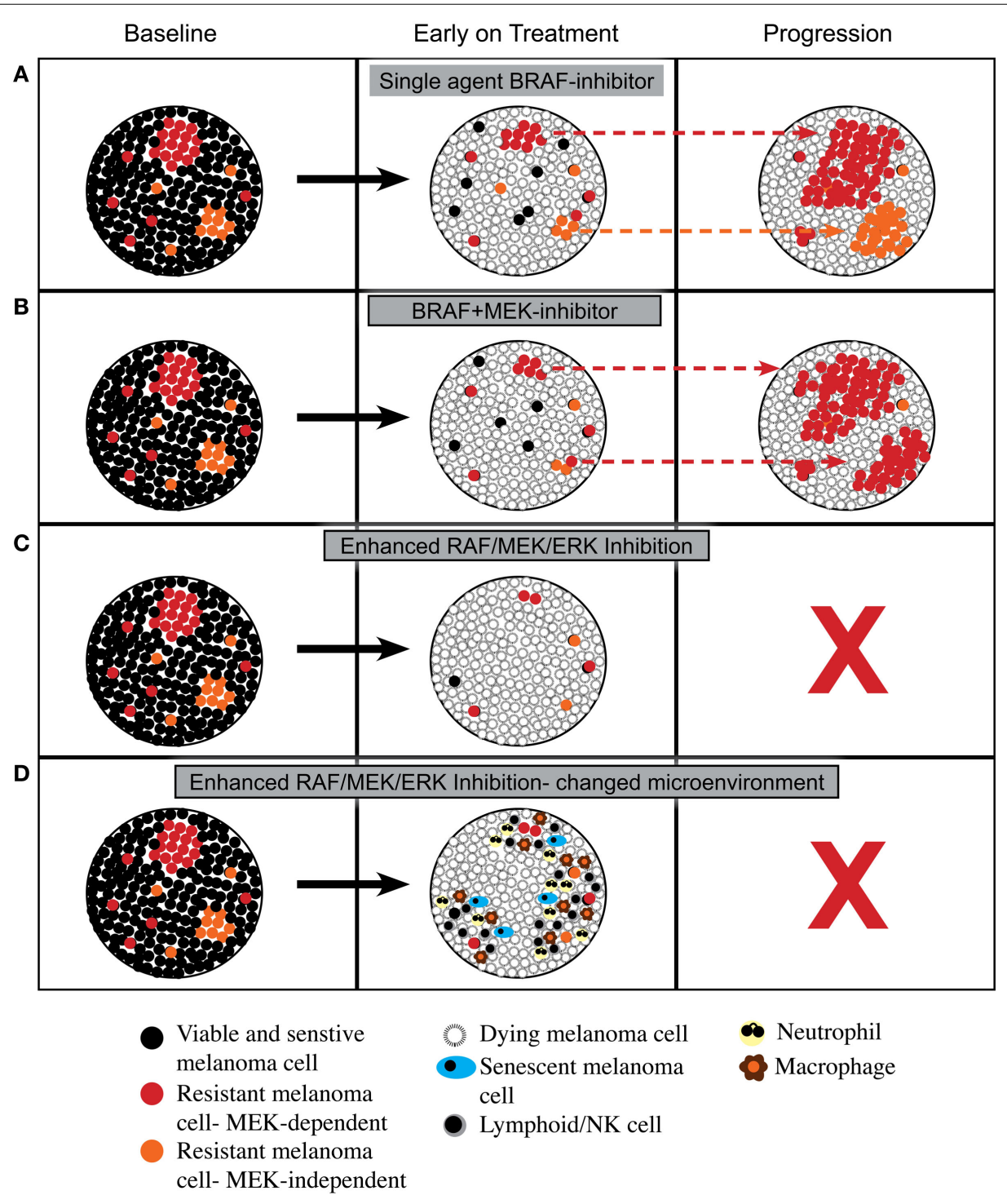

FIGURE 1 | Proposed cellular responses to inhibition of RAF/MEK/ERK signaling. (A) Response to single agent BRAF inhibitor with induction of cell death and then out growth of resistant cells having RAF/MEK/ERK-dependent mechanisms of resistance or RAF/MEK/ERK-independent mechanisms of resistance. (B) Response to combined BRAF and MEK inhibitors with induction of cell death and then out growth of resistant cells dominated by

\section{Strategies to Enhance Inhibition of the RAF/MEK/ERK Pathway}

There are a number of strategies that might improve inhibition of the RAF/MEK/ERK pathway beyond that obtained with continuous exposure to BRAF and MEK inhibitors. Dose, schedule, potency, and inhibiting ERK all have the potential to reduce output from the pathway and result in improved clinical outcomes. A second approach is to inhibit key components of the pathway downstream of ERK. This includes CDK4, pro-apoptotic molecules, such as BIM, and even other signaling networks vital to the outputs of the pathway.
RAF/MEK/ERK-dependent mechanisms of resistance. (C) Response to enhanced inhibition of RAF/MEK/ERK signaling with induction of greater cell death leading to tumor load being below a critical threshold required for outgrowth of resistant cells. (D) Response to enhanced inhibition of RAF/MEK ERK signaling with induction of greater cell death plus a change in tumor microenvironment with influx of leukocytes that prevents emergence of resistance. 
by the use of a schedule of 3 weeks on and 1 week off of the MEK inhibitor cobimetinib when combined with the BRAF inhibitor vemurafenib that is given continuously (44). The intermittent schedule allows a higher dose of cobimetinib to be delivered with likely greater inhibition of pathway output for 3 weeks out of 4 on cobimetinib and vemurafenib when compared to 1 week out of 4 on vemurafenib alone. However, more prolonged interruption of pathway inhibition may provide greater benefits and is being investigated in a clinical trial randomizing patients with advanced BRAF mutant melanoma to continuous or intermittent exposure to dabrafenib and trametinib (NCT02196181).

It is also possible to inhibit the output of the pathway by more effectively inhibiting MEK or possibly by targeting ERK. In the case of trametinib, this can occur through reduction of CRAF/MEK complexes (45). Trametinib, the MEK inhibitor, currently approved in melanoma along with other MEK inhibitors in clinical development are allosteric inhibitors $(45,46)$. In contrast, the ERK inhibitors in clinical development target the ATP binding pocket of the kinase. These properties may influence the output of the pathway when the agents are used in combination possibly due to suppression of feedback mechanisms when compared with MEK inhibitors. Indeed, different allosteric MEK inhibitors can affect feedback to MEK inhibition (45-47) and similar differences may also occur with ERK inhibitors. Furthermore, covalent irreversible inhibitors of ERK have been developed that may further differentiate these agents from MEK inhibitors (48), (NCT02313012). So, further preclinical and clinical data with ERK inhibitors and novel MEK inhibitors are warranted.

A number of approaches can be taken to inhibit the $\mathrm{RAF} / \mathrm{MEK} / \mathrm{ERK}$ pathway downstream of ERK. It is clear that CDK4 activation by ERK is critical to the ability of RAS or RAF to promote cell cycle progression (49). Moreover, genomic changes in the CDK4 regulatory network affect outcomes in patients treated with BRAF inhibitors and can induce resistance $(15,50)$. Interesting CDK4 inhibition can induce irreversible cell cycle arrest and senescence in melanoma cells with BRAF mutations (38). Therefore, combining CDK4 inhibitors with inhibitors of RAF, MEK, and/or ERK is a promising approach that is actively being pursued preclinically and clinically (51).

\section{References}

1. Chapman PB, Hauschild A, Robert C, Haanen JB, Ascierto P, Larkin J, et al. Improved survival with vemurafenib in melanoma with BRAF V600E mutation. N Engl J Med (2011) 364:2507-16. doi:10.1056/NEJMoa1103782

2. Hauschild A, Grob JJ, Demidov LV, Jouary T, Gutzmer R, Millward M, et al. Dabrafenib in BRAF-mutated metastatic melanoma: a multicentre, open-label, phase 3 randomised controlled trial. Lancet (2012) 380:358-65. doi:10.1016/ S0140-6736(12)60868-X

3. Flaherty KT, Robert C, Hersey P, Nathan P, Garbe C, Milhem M, et al. Improved survival with MEK inhibition in BRAF-mutated melanoma. N Engl J Med (2012) 367:107-14. doi:10.1056/NEJMoa1203421

4. Johannessen CM, Boehm JS, Kim SY, Thomas SR, Wardwell L, Johnson LA, et al. COT drives resistance to RAF inhibition through MAP kinase pathway reactivation. Nature (2010) 468:968-72. doi:10.1038/nature 09627

5. Nazarian R, Shi H, Wang Q, Kong X, Koya RC, Lee H, et al. Melanomas acquire resistance to B-RAF(V600E) inhibition by RTK or N-RAS upregulation. Nature (2010) 468:973-7. doi:10.1038/nature09626
Inhibition of the RAF/MEK/ERK pathway can induce apoptosis, principally through activation of the $\mathrm{BH} 3$ alone protein BIM (52, 53), mitochondrial relocalization of BMF (54), and reduction of the anti-apoptotic molecule MCL1 (52). Therefore, the possibility of selectively enhancing the pro-apoptotic affects of RAF/MEK/ERK pathway inhibition through the use of $\mathrm{BH} 3$ mimetics in combination with pathway inhibitors is being investigated in clinical trials (NCT01989585).

Finally, the understanding that ERK can influence other signaling networks offers additional strategies to enhance the biological outcomes of ERK inhibition. Interestingly, protein translation can be regulated by ERK with signaling converging on the EIF4E/4G complex (55) that may also be influenced by the mTOR pathway (56). Therefore, one approach to enhance inhibition of the $\mathrm{RAF} / \mathrm{MEK} / \mathrm{ERK}$ pathway is to combine inhibitors of protein translation or inhibitors of mTORC complexes with BRAF and/or MEK inhibitors.

\section{Conclusion}

The rationale for ongoing investigation of therapeutic strategies to enhance inhibition of the RAF/MEK/ERK pathway is strong. Moreover, the impact of pathway inhibition in the adjuvant setting where there maybe differences in the extent of response of the micro-metastases, or in the microenvironment that emerges following treatment with BRAF and MEK inhibitors necessitates ongoing preclinical and clinical research into therapeutic targeting of the pathway. While it is clear that combination approaches that look at simultaneous or sequential use of immunotherapeutic approaches with agents that target the RAF/MEK/ERK pathway are also a priority, it is not time to divert attention away from the pathway that induces such profound oncogene addiction in melanoma patients whose tumors contain activating mutations in BRAF.

\section{Acknowledgments}

This work was supported by the National Health and Medical Research Council of Australia, the Victorian Cancer Agency, and the Lorenzo and Pamela Galli Charitable Trust.

6. Wagle N, Emery C, Berger MF, Davis MJ, Sawyer A, Pochanard P, et al. Dissecting therapeutic resistance to RAF inhibition in melanoma by tumor genomic profiling. J Clin Oncol (2011) 29:3085-96. doi:10.1200/JCO.2010.33.2312

7. Larkin J, Ascierto PA, Dreno B, Atkinson V, Liszkay G, Maio M, et al. Combined vemurafenib and cobimetinib in BRAF-mutated melanoma. $N$ Engl J Med (2014) 371:1867-76. doi:10.1056/NEJMoa1408868

8. Long GV, Stroyakovskiy D, Gogas H, Levchenko E, De Braud F, Larkin J, et al. Combined BRAF and MEK inhibition versus BRAF inhibition alone in melanoma. N Engl J Med (2014) 371:1877-88. doi:10.1056/NEJMoa1406037

9. Robert C, Karaszewska B, Schachter J, Rutkowski P, Mackiewicz A, Stroiakovski $\mathrm{D}$, et al. Improved overall survival in melanoma with combined dabrafenib and trametinib. N Engl J Med (2015) 372:30-9. doi:10.1056/NEJMoa1412690

10. Su F, Viros A, Milagre C, Trunzer K, Bollag G, Spleiss O, et al. RAS mutations in cutaneous squamous-cell carcinomas in patients treated with BRAF inhibitors. N Engl J Med (2012) 366:207-15. doi:10.1056/NEJMoa1105358

11. Shi H, Moriceau G, Kong X, Lee MK, Lee H, Koya RC, et al. Melanoma whole-exome sequencing identifies (V600E)B-RAF amplification-mediated acquired B-RAF inhibitor resistance. Nat Commun (2012) 3:724. doi:10.1038/ ncomms 1727 
12. Whittaker SR, Theurillat JP, Van Allen E, Wagle N, Hsiao J, Cowley GS, et al. A genome-scale RNA interference screen implicates NF1 loss in resistance to RAF inhibition. Cancer Discov (2013) 3:350-62. doi:10.1158/2159-8290. CD-12-0470

13. Rizos H, Menzies AM, Pupo GM, Carlino MS, Fung C, Hyman J, et al. BRAF inhibitor resistance mechanisms in metastatic melanoma: spectrum and clinical impact. Clin Cancer Res (2014) 20:1965-77. doi:10.1158/1078-0432. CCR-13-3122

14. Shi H, Hong A, Kong X, Koya RC, Song C, Moriceau G, et al. A novel AKT1 mutant amplifies an adaptive melanoma response to BRAF inhibition. Cancer Discov (2014) 4:69-79. doi:10.1158/2159-8290.CD-13-0279

15. Shi H, Hugo W, Kong X, Hong A, Koya RC, Moriceau G, et al. Acquired resistance and clonal evolution in melanoma during braf inhibitor therapy. Cancer Discov (2014) 4:80-93. doi:10.1158/2159-8290.CD-13-0642

16. Van Allen EM, Wagle N, Sucker A, Treacy DJ, Johannessen CM, Goetz $\mathrm{EM}$, et al. The genetic landscape of clinical resistance to RAF inhibition in metastatic melanoma. Cancer Discov (2014) 4:94-109. doi:10.1158/2159-8290. CD-13-0617

17. Trunzer K, Pavlick AC, Schuchter L, Gonzalez R, McArthur GA, Hutson TE, et al. Pharmacodynamic effects and mechanisms of resistance to vemurafenib in patients with metastatic melanoma. J Clin Oncol (2013) 31:1767-74. doi:10. 1200/JCO.2012.44.7888

18. Kwong LN, Boland GM, Frederick DT, Helms TL, Akid AT, Miller JP, et al. Co-clinical assessment identifies patterns of BRAF inhibitor resistance in melanoma. J Clin Invest (2015) 125:1459-70. doi:10.1172/JCI78954

19. Heidorn SJ, Milagre C, Whittaker S, Nourry A, Niculescu-Duvas I, Dhomen $\mathrm{N}$, et al. Kinase-dead BRAF and oncogenic RAS cooperate to drive tumor progression through CRAF. Cell (2010) 140:209-21. doi:10.1016/j.cell.2009.12. 040

20. Poulikakos PI, Zhang C, Bollag G, Shokat KM, Rosen N. RAF inhibitors transactivate RAF dimers and ERK signalling in cells with wild-type BRAF. Nature (2010) 464:427-30. doi:10.1038/nature08902

21. Hatzivassiliou G, Song K, Yen I, Brandhuber BJ, Anderson DJ, Alvarado R, et al. RAF inhibitors prime wild-type RAF to activate the MAPK pathway and enhance growth. Nature (2010) 464:431-5. doi:10.1038/nature08833

22. Solit D, Sawyers CL. Drug discovery: how melanomas bypass new therapy. Nature (2010) 468:902-3. doi:10.1038/468902a

23. Corcoran RB, Ebi H, Turke AB, Coffee EM, Nishino M, Cogdill AP, et al. EGFRmediated re-activation of MAPK signaling contributes to insensitivity of BRAF mutant colorectal cancers to RAF inhibition with vemurafenib. Cancer Discov (2012) 2:227-35. doi:10.1158/2159-8290.CD-11-0341

24. Lito P, Pratilas CA, Joseph EW, Tadi M, Halilovic E, Zubrowski M, et al. Relief of profound feedback inhibition of mitogenic signaling by RAF inhibitors attenuates their activity in BRAFV600E melanomas. Cancer Cell (2012) 22:668-82. doi:10.1016/j.ccr.2012.10.009

25. Prahallad A, Sun C, Huang S, Di Nicolantonio F, Salazar R, Zecchin D, et al. Unresponsiveness of colon cancer to BRAF(V600E) inhibition through feedback activation of EGFR. Nature (2012) 483:100-3. doi:10.1038/nature10868

26. Lito P, Rosen N, Solit DB. Tumor adaptation and resistance to RAF inhibitors. Nat Med (2013) 19:1401-9. doi:10.1038/nm.3392

27. Long GV, Fung C, Menzies AM, Pupo GM, Carlino MS, Hyman J, et al. Increased MAPK reactivation in early resistance to dabrafenib/trametinib combination therapy of BRAF-mutant metastatic melanoma. Nat Commun (2014) 5:5694. doi:10.1038/ncomms6694

28. Wagle N, Van Allen EM, Treacy DJ, Frederick DT, Cooper ZA, Taylor-Weiner A, et al. MAP kinase pathway alterations in BRAF-mutant melanoma patients with acquired resistance to combined RAF/MEK inhibition. Cancer Discov (2014) 4:61-8. doi:10.1158/2159-8290.CD-13-0631

29. Moriceau G, Hugo W, Hong A, Shi H, Kong X, Yu CC, et al. Tunablecombinatorial mechanisms of acquired resistance limit the efficacy of BRAF/MEK cotargeting but result in melanoma drug addiction. Cancer Cell (2015) 27:240-56. doi:10.1016/j.ccell.2014.11.018

30. Bollag G, Hirth P, Tsai J, Zhang J, Ibrahim PN, Cho H, et al. Clinical efficacy of a RAF inhibitor needs broad target blockade in BRAF-mutant melanoma. Nature (2010) 467:596-9. doi:10.1038/nature09454

31. Zipser MC, Eichhoff OM, Widmer DS, Schlegel NC, Schoenewolf NL, Stuart $\mathrm{D}$, et al. A proliferative melanoma cell phenotype is responsive to RAF/MEK inhibition independent of BRAF mutation status. Pigment Cell Melanoma Res (2011) 24:326-33. doi:10.1111/j.1755-148X.2010.00823.x
32. Caramel J, Papadogeorgakis E, Hill L, Browne GJ, Richard G, Wierinckx A, et al A switch in the expression of embryonic EMT-inducers drives the development of malignant melanoma. Cancer Cell (2013) 24:466-80. doi:10.1016/j.ccr.2013. 08.018

33. Haq R, Shoag J, Andreu-Perez P, Yokoyama S, Edelman H, Rowe GC, et al. Oncogenic BRAF regulates oxidative metabolism via PGC1alpha and MITF. Cancer Cell (2013) 23:302-15. doi:10.1016/j.ccr.2013.02.003

34. Li FZ, Dhillon AS, Anderson RL, McArthur G, Ferrao PT. Phenotype switching in melanoma: implications for progression and therapy. Front Oncol (2015) 5:31. doi:10.3389/fonc.2015.00031

35. Wilmott JS, Long GV, Howle JR, Haydu LE, Sharma RN, Thompson JF, et al. Selective BRAF inhibitors induce marked T-cell infiltration into human metastatic melanoma. Clin Cancer Res (2012) 18:1386-94. doi:10.1158/ 1078-0432.CCR-11-2479

36. Frederick DT, Piris A, Cogdill AP, Cooper ZA, Lezcano C, Ferrone $\mathrm{CR}$, et al. BRAF inhibition is associated with enhanced melanoma antigen expression and a more favorable tumor microenvironment in patients with metastatic melanoma. Clin Cancer Res (2013) 19:1225-31. doi:10.1158/ 1078-0432.CCR-12-1630

37. Knight DA, Ngiow SF, Li M, Parmenter T, Mok S, Cass A, et al. Host immunity contributes to the anti-melanoma activity of BRAF inhibitors. J Clin Invest (2013) 123:1371-81. doi:10.1172/JCI66236

38. Haferkamp S, Borst A, Adam C, Becker TM, Motschenbacher S, Windhovel $\mathrm{S}$, et al. Vemurafenib induces senescence features in melanoma cells. J Invest Dermatol (2013) 133:1601-9. doi:10.1038/jid.2013.6

39. McArthur GA, Callahan J, Ribas A, Gonzalez R, Pavlick AC, Hamid O, et al. Metabolic tumor burden for prediction of overall survival following combined BRAF/MEK inhibition in patients with advanced BRAF mutant melanoma. $J$ Clin Oncol (2014) 32(suppl; abstr 9006^):5s.

40. Parmenter TJ, Kleinschmidt M, Kinross KM, Bond ST, Li J, Kaadige MR, et al. Response of BRAF mutant melanoma to BRAF inhibition is mediated by a network of transcriptional regulators of glycolysis. Cancer Discov (2014) 4(4):423-33. doi:10.1158/2159-8290.CD-13-0440

41. Druker BJ, Talpaz M, Resta DJ, Peng B, Buchdunger E, Ford JM, et al. Efficacy and safety of a specific inhibitor of the BCR-ABL tyrosine kinase in chronic myeloid leukemia. N Engl J Med (2001) 344:1031-7. doi:10.1056/ NEJM200104053441401

42. Shah NP, Kasap C, Weier C, Balbas M, Nicoll JM, Bleickardt E, et al. Transient potent BCR-ABL inhibition is sufficient to commit chronic myeloid leukemia cells irreversibly to apoptosis. Cancer Cell (2008) 14:485-93. doi:10.1016/j.ccr. 2008.11.001

43. Das Thakur M, Salangsang F, Landman AS, Sellers WR, Pryer NK, Levesque $\mathrm{MP}$, et al. Modelling vemurafenib resistance in melanoma reveals a strategy to forestall drug resistance. Nature (2013) 494:251-5. doi:10.1038/nature11814

44. Ribas A, Gonzalez R, Pavlick A, Hamid O, Gajewski TF, Daud A, et al. Combination of vemurafenib and cobimetinib in patients with advanced BRAF(V600)mutated melanoma: a phase 1b study. Lancet Oncol (2014) 15:954-65. doi:10. 1016/S1470-2045(14)70301-8

45. Lito P, Saborowski A, Yue J, Solomon M, Joseph E, Gadal S, et al. Disruption of CRAF-mediated MEK activation is required for effective MEK inhibition in KRAS mutant tumors. Cancer Cell (2014) 25:697-710. doi:10.1016/j.ccr.2014. 03.011

46. Ishii N, Harada N, Joseph EW, Ohara K, Miura T, Sakamoto H, et al. Enhanced inhibition of ERK signaling by a novel allosteric MEK inhibitor, CH5126766, that suppresses feedback reactivation of RAF activity. Cancer Res (2013) 73:4050-60. doi:10.1158/0008-5472.CAN-12-3937

47. Hatzivassiliou G, Haling JR, Chen H, Song K, Price S, Heald R, et al. Mechanism of MEK inhibition determines efficacy in mutant KRAS- versus BRAF-driven cancers. Nature (2013) 501:232-6. doi:10.1038/nature12441

48. Liu Q, Sabnis Y, Zhao Z, Zhang T, Buhrlage SJ, Jones LH, et al. Developing irreversible inhibitors of the protein kinase cysteinome. Chem Biol (2013) 20:146-59. doi:10.1016/j.chembiol.2012.12.006

49. Drosten M, Dhawahir A, Sum EY, Urosevic J, Lechuga CG, Esteban LM, et al. Genetic analysis of Ras signalling pathways in cell proliferation, migration and survival. EMBO J (2010) 29:1091-104. doi:10.1038/emboj.2010.7

50. Nathanson KL, Martin AM, Wubbenhorst B, Greshock J, Letrero R, D’Andrea $\mathrm{K}$, et al. Tumor genetic analyses of patients with metastatic melanoma treated with the BRAF inhibitor dabrafenib (GSK2118436). Clin Cancer Res (2013) 19:4868-78. doi:10.1158/1078-0432.CCR-13-0827 
51. Lee B, Sandhu S, McArthur G. Cell cycle control as a promising target in melanoma. Curr Opin Oncol (2015) 27:141-50. doi:10.1097/CCO. 0000000000000159

52. Wang YF, Jiang CC, Kiejda KA, Gillespie S, Zhang XD, Hersey P. Apoptosis induction in human melanoma cells by inhibition of MEK is caspaseindependent and mediated by the Bcl-2 family members PUMA, Bim, and Mcl1. Clin Cancer Res (2007) 13:4934-42. doi:10.1158/1078-0432.CCR-07-0665

53. Cragg MS, Jansen ES, Cook M, Harris C, Strasser A, Scott CL. Treatment of B-RAF mutant human tumor cells with a MEK inhibitor requires Bim and is enhanced by a BH3 mimetic. J Clin Invest (2008) 118:3651-9. doi:10.1172/ JCI35437

54. VanBrocklin MW, Verhaegen M, Soengas MS, Holmen SL. Mitogen-activated protein kinase inhibition induces translocation of Bmf to promote apoptosis in melanoma. Cancer Res (2009) 69:1985-94. doi:10.1158/0008-5472. CAN-08-3934

55. Boussemart L, Malka-Mahieu H, Girault I, Allard D, Hemmingsson O, Tomasic $\mathrm{G}$, et al. eIF4F is a nexus of resistance to anti-BRAF and anti-MEK cancer therapies. Nature (2014) 513:105-9. doi:10.1038/nature13572
56. Corcoran RB, Rothenberg SM, Hata AN, Faber AC, Piris A, Nazarian RM, et al. TORC1 suppression predicts responsiveness to RAF and MEK inhibition in BRAF-mutant melanoma. Sci Transl Med (2013) 5:196ra198. doi:10.1126/ scitranslmed. 3005753

Conflict of Interest Statement: Prof. Grant A. McArthur is a compensated consultant for Provectus and an uncompensated consultant for Bristol-Myers Squibb, GlaxoSmithKline, Amgen, Novartis, and Roche-Genentech, and receives research funding from Pfizer, Celgene, and Ventana.

Copyright $(\odot 2015$ McArthur. This is an open-access article distributed under the terms of the Creative Commons Attribution License (CC BY). The use, distribution or reproduction in other forums is permitted, provided the original author(s) or licensor are credited and that the original publication in this journal is cited, in accordance with accepted academic practice. No use, distribution or reproduction is permitted which does not comply with these terms. 